\title{
International Linear Collider damping ring lattice design based on modified FODO arc cells
}

\author{
Y.P. Sun, ${ }^{1,2, *}$ J. Gao, ${ }^{2}$ Z. Y. Guo, ${ }^{1}$ and W. S. Wan ${ }^{3}$ \\ ${ }^{1}$ Key Laboratory of Heavy Ion Physics, Ministry of Education \& School of Physics, Peking University, Beijing 100871, China \\ ${ }^{2}$ Institute of High Energy Physics, Chinese Academy of Science, Beijing 100049, China \\ ${ }^{3}$ Lawrence Berkeley National Laboratory, Berkeley, California 94720, USA \\ (Received 2 October 2007; published 23 June 2008)
}

\begin{abstract}
In this paper, we present a new lattice design for the International Linear Collider (ILC) positron damping ring. The same lattice could be used also for the electron damping ring. This lattice is based on modified FODO arc cells, and has a freely tunable momentum compaction factor from $2 \times 10^{-4}$ to $6 \times$ $10^{-4}$. In comparison with the baseline design which uses the theoretical minimum emittance arc cells, the advantages of this design are: freely tunable momentum compaction factor; smaller number of quadrupole and sextupole magnets; larger dynamic aperture; simplified layout and lower cost. The design principles and analysis techniques used in this paper to change the momentum compaction factor by a large amount while keeping a very low emittance and large dynamic aperture are very important for the ILC damping ring; at the same time it could be very useful for any high bunch density storage ring.
\end{abstract}

DOI: 10.1103/PhysRevSTAB.11.061001

PACS numbers: 29.20.db, 29.27.Bd, 21.10.Tg

\section{INTRODUCTION}

Damping ring lattices are widely designed and studied during the linear collider design process [1-8]. The damping rings are necessary to accept positron and electron beams with large energy spread and transverse emittance, coming from the sources, and to produce beams with very low emittance for the fulfillment of the linear collider's high luminosity goal. Roughly thousands of bunches will be stored in the damping ring, with each bunch injected and extracted individually.

During the baseline design and reference design phase, as all the vacuum elements have not been technically designed, the total impedance can only be estimated with the previous experiences. So the momentum compaction factor of the damping ring is better to be tunable in a proper range, for the safe running of the damping ring under some single bunch collective effects threshold. In order to achieve these requirements, the damping rings should have proper circumference, very fast injection/extraction kickers, large acceptance, fast damping time, and tunable momentum compaction factor.

The recommendation for the International Linear Collider (ILC) damping ring baseline lattice design is based on the studies of seven different reference lattices. These seven reference designs cover different circumference, layout, beam energy, and arc cell structures. In 2005, a $6.7 \mathrm{~km}$ ring composed of theoretical minimum emittance (TME) arc cells (called OCS lattice), with $5 \mathrm{GeV}$ beam energy, was selected as the baseline design on the basis of cost and technical risk [9]. Up to now, the ILC damping ring baseline design has its newest version OCS8 in design and optimization process [10]. The circumference, the

\footnotetext{
*ypsun@ihep.ac.cn
}

momentum compaction factor, and also the dynamic aperture of the OCS8 lattice need to be optimized further.

Our new damping ring optics design based on modified FODO arc cells initiated from the end of 2005. This configuration is not included in the seven reference lattices mentioned above. It has several key advantages over the baseline design which will be presented in the following sections.

\section{GLOBAL CONSIDERATIONS AND THE LAYOUT}

Similar to the baseline design, our damping ring design includes one positron ring and one electron ring which will be housed in a single central tunnel of ILC. The circumference is selected to be $6476.439 \mathrm{~m}$, which is according to the newest constraints from the downstream bunch compressor systems. Our damping ring design is composed of four arc sections and four straight sections, with two straight sections to accommodate the superconducting rf stations and superconducting damping wigglers, and the other two to accommodate the injection and extraction elements (also chicane). The FODO cells are used in both the arc sections and straight sections. The layout of our FODO damping ring design is shown in Fig. 1 with the beams counter-rotating in the two rings.

The damping ring must be capable of reducing the emittances of the incoming positron and electron bunches to the required level within a $200 \mathrm{~ms}$ interval between machine pulses, which is constrained by the $5 \mathrm{~Hz}$ machine repetition rate. Consequently, the damping ring should have very low normalized emittance and very fast damping time, which are mainly achieved by the long wiggler magnets installed in damping rings. However, the arc cell is also important for the emittance and damping time. Write the equilibrium beam emittance in a separated func- 


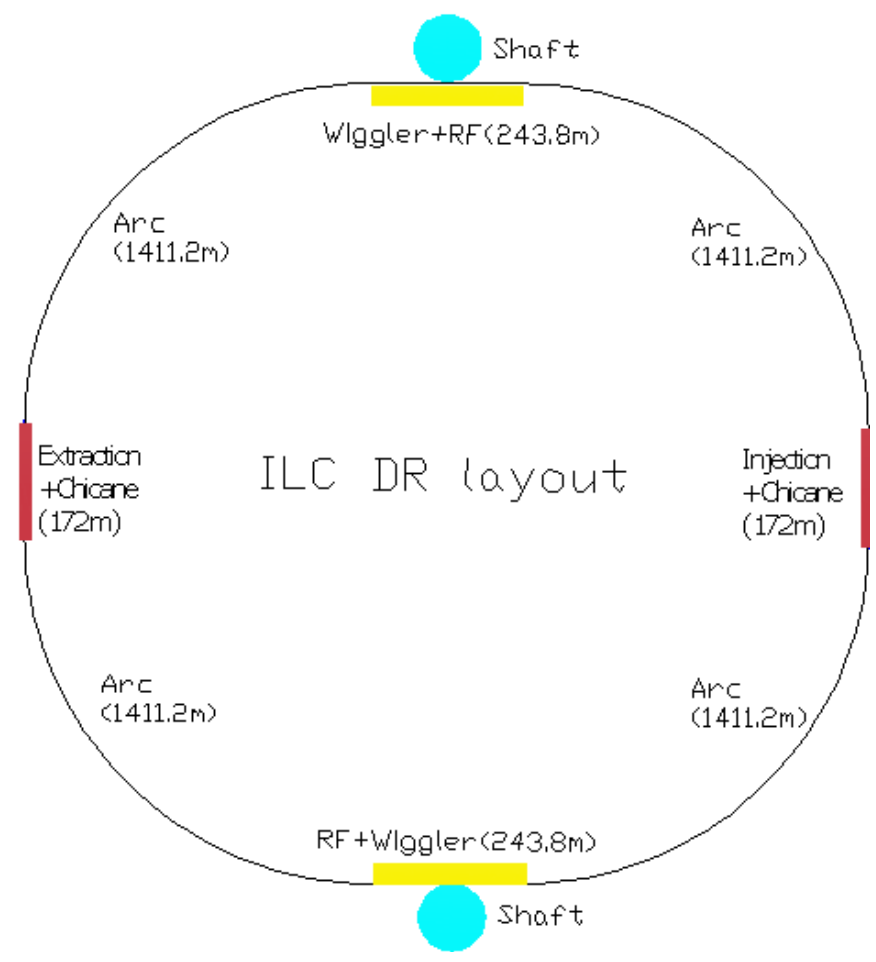

FIG. 1. (Color) FODO damping ring layout.

tions storage ring as

$$
\varepsilon_{x, \min }=\frac{F}{12 \sqrt{15}} C_{q} \gamma^{2} \frac{\theta^{3}}{J_{x}}
$$

where $C_{q}=3.84 \times 10^{-13} \mathrm{~m}, \theta$ is the bending angle in each dipole magnet, and $F$ is a dimensionless factor depending on the lattice structure of the arc cell. Studies show that the minimum emittance can be achieved for TME arc cells and corresponds to a value of $F=1[5,11]$.
The normal FODO cell has $F$ roughly equal to 100 . However, in a modified FODO cell the $F$ value can be decreased a lot, so that the modified FODO cell can be used as the arc cell of the damping ring and fulfill the equilibrium emittance goal. In our damping ring design, FODO cells are used in the arc section, first with the aim to decrease the total quadrupole number and sextupole number by one third. At the same time, for the tunable momentum compaction factor requirements, FODO arc cells are much more easily tunable, compared with the TME arc cells. At last, with a storage ring composed of FODO cells it has been easily achieved a large dynamic aperture, less sensitive to the high-order magnet errors with respect to the TME lattice.

With all the above considerations, modified FODO arc cells are used to compose our damping ring lattice in order to achieve the following design goals: (1) a freely tunable momentum compaction factor between $2 \times 10^{-4}$ and $6 \times$ $10^{-4}$; (2) a betatron function lower than $55 \mathrm{~m}$, constrained by the vacuum chamber size; (3) a good dynamic aperture for all the momentum compaction factor lattices, which constrains the Twiss parameters and betatron phase advances at the sextupole magnets locations. The following parameters have been scanned and studied: (1) the total arc cell number between 120 and 240; (2) the length of one single arc cell between $20 \mathrm{~m}$ and $40 \mathrm{~m}$; (3) in one arc cell, the length of the short drift between 1 and $3 \mathrm{~m}$; (4) the phase advance of one arc cell between 60/60 degree and 90/90 degree.

At last, 184 arc cells are selected to compose the damping ring, with each arc cell length as $29.4 \mathrm{~m}$; the length of the short drift is selected to be $2 \mathrm{~m}$. The momentum compaction change is achieved by changing the phase advance per cell and retuning the dispersion suppressor section. The standard phase advance of the arc cell is

TABLE I. The main parameters of three critical damping ring running modes.

\begin{tabular}{lccc}
\hline \hline \multicolumn{1}{c}{ Parameter } & $\alpha_{P}=2 \times 10^{-4}$ & $\alpha_{P}=4 \times 10^{-4}$ & $\alpha_{P}=6 \times 10^{-4}$ \\
\hline Circumference [m] & 6476.439 & 6476.439 & 6476.439 \\
Harmonic number & 14042 & 14042 & 14042 \\
Energy [GeV] & 5 & 5 & 5 \\
Arc cell & FODO & FODO & FODO \\
Tune & $58.29 / 57.25$ & $48.28 / 47.24$ & $41.29 / 40.25$ \\
Natural chromaticity & $-74 /-73$ & $-54 /-55$ & $-48 /-49$ \\
Momentum compaction $\left[10^{-4}\right]$ & 2 & 4 & 6 \\
Transverse damping time $[\mathrm{ms}]$ & $25 / 25$ & $25 / 25$ & $25 / 25$ \\
Normalized natural emittance [mm mrad] & 3.36 & 4.2 & 5.4 \\
rf voltage [MV] & 15 & 22 & 31 \\
Synchrotron tune & 0.038 & 0.061 & 0.091 \\
Synchrotron phase [ ${ }^{\circ}$ ] & 145 & 157 & 164 \\
rf frequency [MHz] & 650 & 650 & 650 \\
rf acceptance [\%] & 1.21 & 1.48 & 1.65 \\
Natural bunch length [mm] & 9 & 9 & 9 \\
Natural energy spread [10 & & & \\
\hline \hline
\end{tabular}


TABLE II. The parameters of the magnets.

\begin{tabular}{lcccc}
\hline \hline Element & $\begin{array}{c}\text { Length } \\
{[\mathrm{m}]}\end{array}$ & $\begin{array}{c}\text { Field or } \\
\text { gradient }\end{array}$ & $\begin{array}{c}\text { Aperture } \\
{[\mathrm{m}]}\end{array}$ & $\begin{array}{c}\text { Pole-tip } \\
\text { field [T] }\end{array}$ \\
\hline Dipole & 2 & $0.2246 \mathrm{~T}$ & 0.06 & 0.2246 \\
Quadrupole & 0.3 & $10 \mathrm{~T} / \mathrm{m}$ & 0.06 & 0.3 \\
Sextupole & 0.25 & $17.67 \mathrm{~T} / \mathrm{m}^{2}$ & 0.06 & 0.00796 \\
\hline \hline
\end{tabular}

$72 / 72$ degree, with the momentum compaction factor as $4 \times 10^{-4}$; the 60/60 degree and 90/90 degree arc cell phase advance is according to the momentum compaction factor $6 \times 10^{-4}$ and $2 \times 10^{-4}$, respectively, while the whole lattice can be tuned freely between these two sets, by only changing the quadrupole power supply. The parameters of these three configurations are shown in Table I.

For the technical considerations, we will briefly outline the general requirements and specifications. The rf frequency is $650 \mathrm{MHz}$, or one-half of the main linac rf frequency, and the voltage is between 15 and $31 \mathrm{MV}$. The corresponding equilibrium rms bunch length is $9 \mathrm{~mm}$. The injection/extraction is achieved by using two septums and a large number of weak fast stripline kickers, with the effective kicker pulse width less than twice the bunch spacing. The bunch separation is much longer in the ILC main linac accelerator than in the damping rings, so the bunch must be injected and extracted individually, without affecting the emittance and stability of the other stored bunches. The damping wigglers are superconducting and all the other magnets are room temperature. The main parameters of the damping wigglers are as follows: the peak field $1.6 \mathrm{~T}$, the pole length $0.1 \mathrm{~m}$, the period $0.4 \mathrm{~m}$, and the total length $196 \mathrm{~m}$. The parameters of the dipoles, quadrupoles, and sextupoles in our damping ring lattice design are listed in Table II. The vacuum chamber material is aluminum and the cross section is circular.

In the baseline damping ring design, there are 747 quadrupoles and 504 sextupoles in total. In our damping ring design, the total quadrupole number is 448 and the total sextupole number is 368 , which means that the total cost of the magnets will be largely decreased.

\section{OPTICS DESIGN}

There are 184 arc cells in all in our damping ring design, therefore each dipole magnet provides a bending angle of $2 \pi / 368$ for the beam. The FODO arc cell length is selected as $29.4 \mathrm{~m}$ and the standard phase advance per arc cell is $72^{\circ} / 72^{\circ}$ for the horizontal and vertical betatron motion, respectively. Equations (2) and (3) specify the maximum and minimum values of the beta functions and horizontal dispersion functions in one normal FODO cell [12]. For the modified FODO cells, these values shall be modified a little, especially the horizontal dispersion function:

$$
\beta^{ \pm}=\frac{L_{P}\left(1 \pm \sin \frac{\mu}{2}\right)}{\sin \mu}
$$

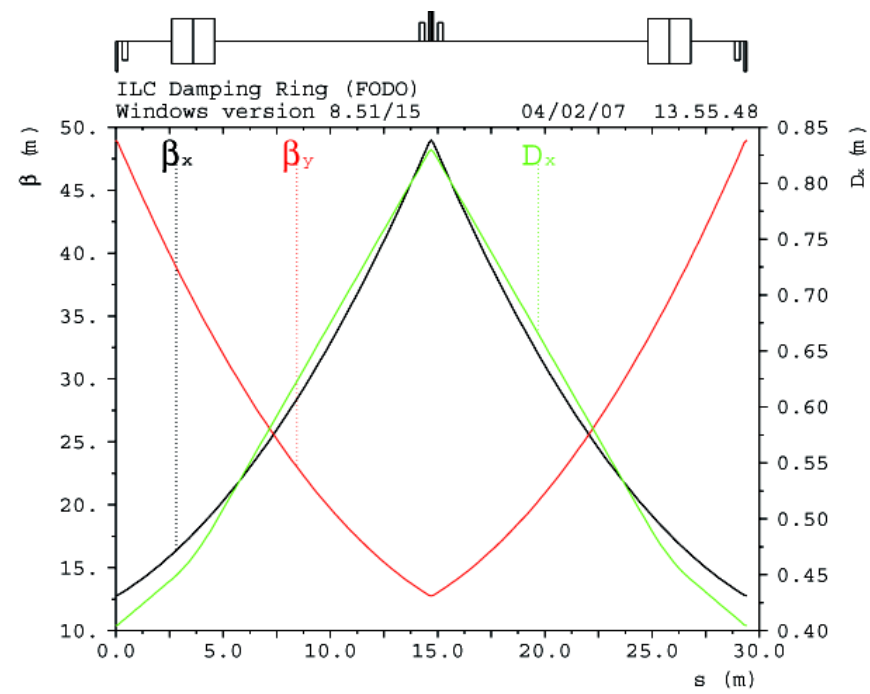

FIG. 2. (Color) Lattice functions in the 72/72 degree arc cell.

$$
D^{ \pm}=\frac{L_{P} \phi\left(1 \pm \frac{1}{2} \sin \frac{\mu}{2}\right)}{4 \sin ^{2} \frac{\mu}{2}}
$$

where $\beta^{+}$and $\beta^{-}$are the maximum and minimum value of the beta functions, $D^{+}$and $D^{-}$are the maximum and minimum value of the horizontal dispersion function, $L_{P}$ is the length of the cell, $\mu$ is the phase advance of the cell, and $\varphi$ is the bending angle in one arc cell. To have smaller rms dispersion value, the length of the short drift is adjusted to be $2 \mathrm{~m}$. The resulting horizontal dispersion can fulfill the requirements of the equilibrium emittance, the momentum compaction factor, and chromaticity correction sextupoles need.

For the $72 / 72$ degree phase advance case, the lattice functions in an arc cell are shown in Fig. 2. To tune the phase advance to another value between the 60/60 degree and the 90/90 degree, only the quadrupoles' strength needs to be changed.

For the design of the dispersion suppressor (90 degree arc cell case) which is placed at the exit of each arc section, we explore the technique which adds one extra arc cell outside the last normal arc cell. The bending angle of these two cells is modified according to [12]

$$
\begin{gathered}
\varphi_{1}=\varphi \cdot\left(1-\frac{1}{4 \sin ^{2} \frac{\mu}{2}}\right) \\
\varphi_{2}=\frac{\varphi}{4 \sin ^{2} \frac{\mu}{2}},
\end{gathered}
$$

where $\varphi$ is the bending angle of the normal arc cell which is 90 degrees, $\varphi_{1}$ and $\varphi_{2}$ are bending angles of the FODO cell on the side where $D \neq 0$ and $D=0$. In this kind of dispersion suppressor design, the betatron oscillations are not disturbed in both styles of dispersion suppressor, which are good for the matching of other sections and also the chromaticity correction. It can start at horizontally focus- 


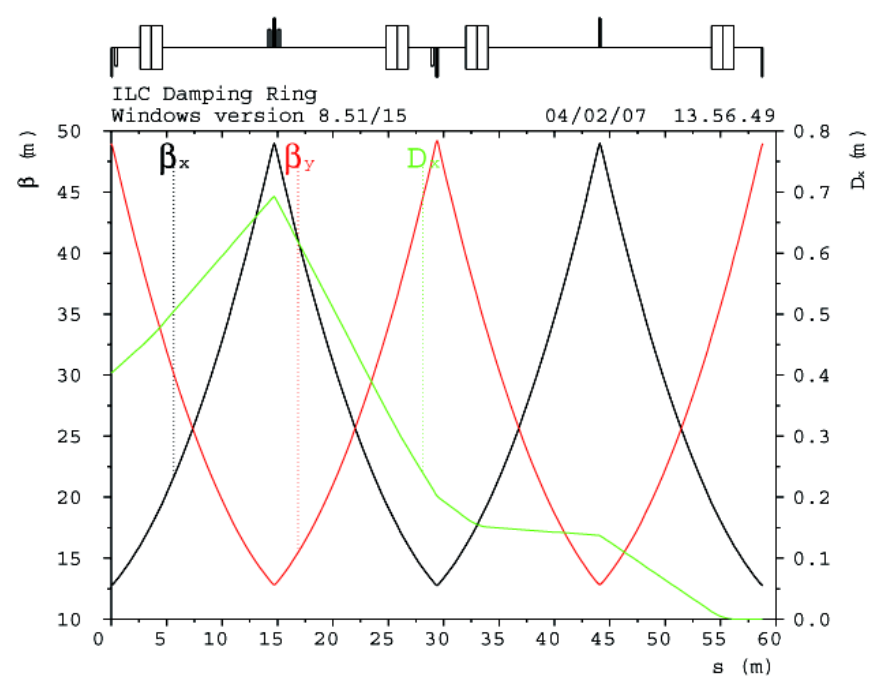

FIG. 3. (Color) Lattice functions in the 90/90 degree dispersion suppressor.

ing or defocusing quadrupoles. For other cases with arc cell phase advance between 60/60 degrees and 90/90 degrees, the dipole strength is the same with the 90 degree case mentioned above (so the ring geometry is not changed), and the strength of the quadrupoles in the dispersion suppressor section is rematched to achieve a good matching of dispersion to zero at exit. The optics design of the disper-

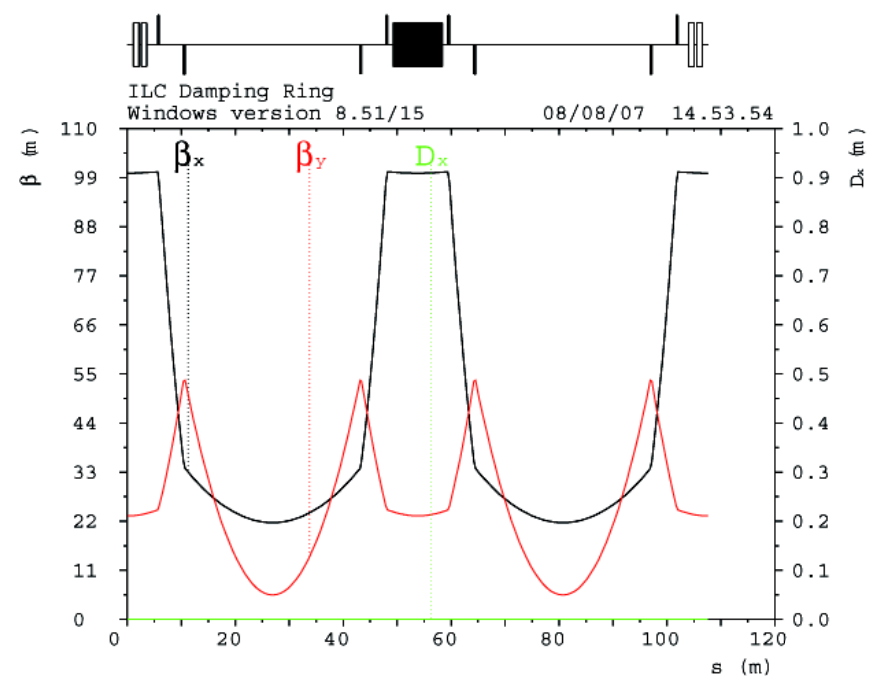

FIG. 4. (Color) Lattice functions in the injection/extraction sections. sion suppressor is shown in Fig. 3 for the case of the 90/90 degree phase advance.

The newest ILC design requires the damping ring to be placed at the center of the whole collider, with the injection and extraction sections at the two opposite sides of the damping ring. The injection and extraction optics should have the same design, which uses 2 septums and 21 stripline kickers [13]. The kicker modules are $50 \Omega$ stripline structures inside the vacuum pipe, each $30 \mathrm{~cm}$ long, operating at the voltage of $22 \mathrm{kV}$. Twenty-one such modules are needed for the injection of the beam and 11 are used for extraction. The thin septum magnet has a $0.73 \mathrm{~T}$ magnetic field and the thick septum magnet's field is $1.08 \mathrm{~T}$, which are modeled after the Argonne APS injection septa. The optics design of the injection/extraction section is shown in Fig. 4. This section uses two periodic cells, with the total horizontal phase advance matched to be 180 degrees. The horizontal phase advance between the septum magnet and the stripline kickers is 90 degrees, and the alpha function is designed to be zero at the both exit point. In comparison with the previous design [6], the horizontal beta function at the stripline kicker is increased from 17 to $100 \mathrm{~m}$, and the horizontal beta function at the septum magnet is increased from 40 to $100 \mathrm{~m}$. The advantage of this configuration is to reduce the ratio between septum thickness and injected beam size, reducing the kickers strength, and to allow placing all the stripline kickers in the same drift.

Thermal changes and ground motion effects could affect the damping ring circumference. As stated before, the rf frequency of the damping ring is locked with the rf frequency of the main linac accelerator, which is why we have to add chicanes into the ILC damping ring lattice [14]. A chicane is a kind of special optics section composed of dipoles and drifts. The schematic layout of the compact "zigzag" chicane is shown in Fig. 5. In order to provide $10^{-6}$ adjustment ability on the damping ring circumference during the commissioning process, the chicane need to have $\pm 7 \mathrm{~mm}$ adjustment ability.

Four zigzag chicanes are placed in the injection/extraction straight section. For a single chicane, the path length adjustment is $\pm 2 \theta^{2}\left(l_{c}+0.5 l_{B}\right)$, where $\theta$ is the bending angle of the first dipole, $l_{c}$ is the drift length between the inner and outer dipole, and $l_{B}$ is the half length of the outer dipole. Under careful consideration, we take $l_{c}=7 \mathrm{~m}$, $l_{B}=1 \mathrm{~m}, \theta=0.0108 \mathrm{rad}$. The magnetic strength of the dipole is $0.4 \mathrm{~T}$. The injection/extraction optics with chicanes added in is shown in Fig. 6. The extra quantum

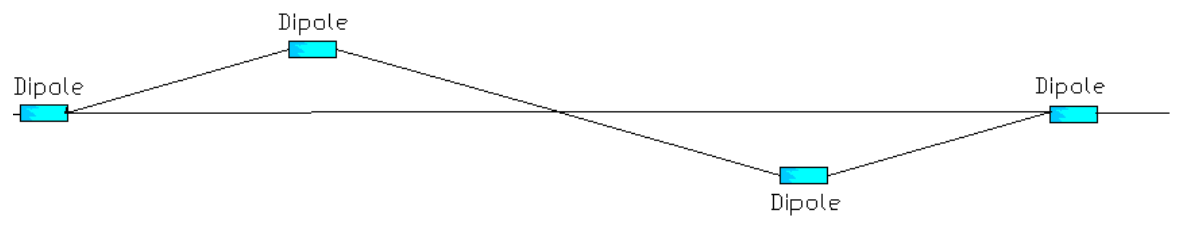

FIG. 5. (Color) Schematic layout of the compact zigzag chicane. 


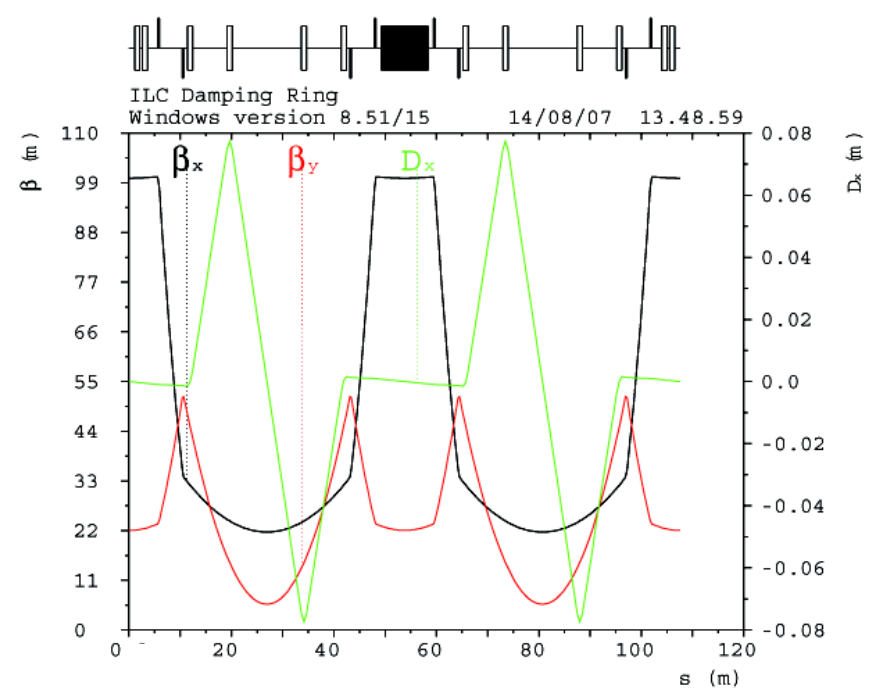

FIG. 6. (Color) Injection/extraction optics with chicane.

excitation generated by chicanes will increase the emittance of the damping ring. Preliminary calculation shows that the equilibrium emittance will increase by $9.2 \%$.

The optics design of the straight section which is used to accommodate rf cavity and damping wigglers is the same with the baseline design [9]. For the matching sections between the dispersion suppressor and the straight sections, we roughly use 4 to 6 quadrupoles to match the Twiss parameters in both sides, and have reasonable beta functions. What should be mentioned here is that the length of the matching sections can be adjusted in a small range in order to get a reasonable circumference and fill patterns [15] in the next design phase. By tuning the quadrupole strength in the damping ring, the momentum compaction factor can be tuned freely between $2 \times 10^{-4}$ and $6 \times 10^{-4}$.

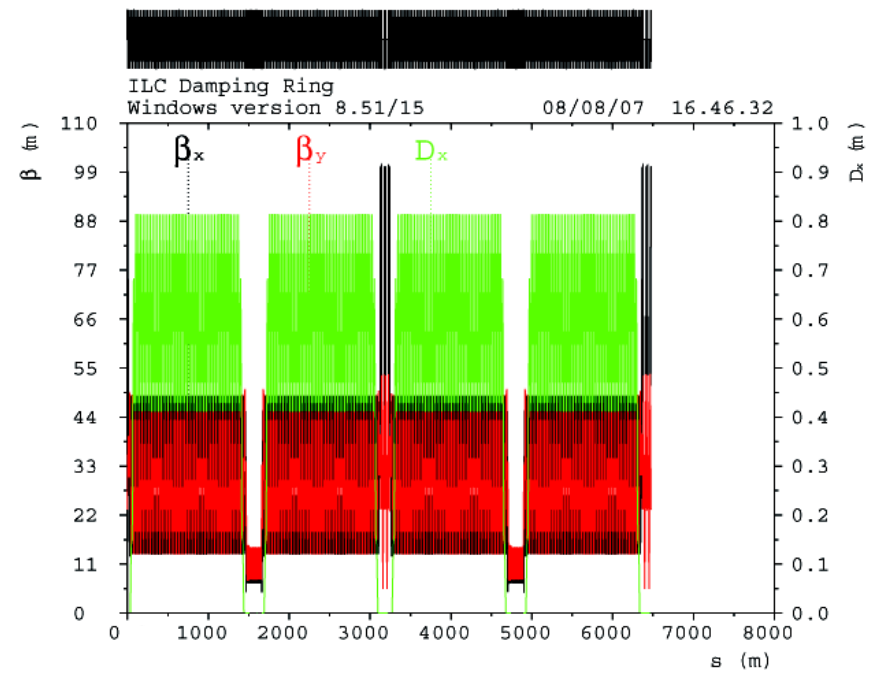

FIG. 7. (Color) Twiss parameters for the whole ring, with momentum compaction factor $4 \times 10^{-4}$.
The Twiss parameters for the whole ring with momentum compaction factor $4 \times 10^{-4}$ are shown in Fig. 7 .

\section{OPTIMIZATION OF THE DYNAMIC APERTURE}

Dynamic aperture should ensure efficient acceptance of the large emittance beam from the positron source, in fact the required value of 3 times the injected beam size corresponds to nearly 50 times the equilibrium beam size. During the damping ring design process, the natural chromaticity generated by the straight sections is controlled to be as small as possible. Two groups of interlaced sextupoles are placed in every normal arc cell, to correct the first-order chromaticity to zero. The drift between the sextupole and its adjacent quadrupole has a length of $0.2 \mathrm{~m}$, to have a larger horizontal and vertical beta function difference at the sextupole location. At the same time, the arc cell is designed to have a larger horizontal dispersion function at the sextupole location, in order to decrease the sextupole strength and have a better dynamic aperture. Regarding the phase advance of the straight sections, they are matched to be near integer of half integer, to increase the symmetry of the whole ring and decrease the higher order resonances caused by the sextupoles. The experiences show that these kinds of methods are really useful.

Based on the dynamic aperture tracking and frequency map analysis (FMA) results, the phase advance of every normal arc cell is also tuned a little, in the range of $\pm 1 \%$. The aim is to have the right phase at the sextupole locations with which the nonlinear effects caused by chromaticity sextupoles cancel each other best. The fractional part of the damping ring's total tune is adjusted to be near $(0.29,0.25)$ which has been proven to be comparatively good. At the same time, the horizontal tune is adjusted to be 1 larger than the vertical tune. After optimization, the achieved dynamic aperture without magnet errors is larger than 10-12 times injected positron bunch size for the $6 \times$ $10^{-4}$ momentum compaction mode, with $\pm 1 \%$ energy spread. For the $4 \times 10^{-4}$ and $2 \times 10^{-4}$ momentum compaction modes, the achieved dynamic apertures are 8-10 times and 4.3-6 times injected positron bunch size, respectively.

Compared with the previous work that has been done on damping ring lattice design based on FODO arc cells [8], our damping ring lattice design has almost been optimized to get the best dynamic aperture that was ever achieved. At the same time, the dynamic aperture of this design is a little better than the baseline design which is based on TME arc cells [10]. However, it is believed that there is still margin in the dynamic aperture optimization on this damping ring lattice.

Add the high-order magnet errors listed in Table III into the dynamic aperture tracking process (take 10 seeds averaged), then track one single particle for 1000 turns in the 
TABLE III. The high-order magnet errors (Sys stands for systematic errors, Ran stands for random errors, and $\Phi$ is the angle).

\begin{tabular}{|c|c|c|c|c|c|c|c|c|c|}
\hline \multirow{3}{*}{$\begin{array}{l}\text { Radius } \\
\text { Error type }\end{array}$} & \multicolumn{3}{|c|}{ Dipole $\left(1 \times 10^{-4}\right)$} & \multicolumn{3}{|c|}{ Quadrupole $\left(1 \times 10^{-4}\right)$} & \multicolumn{3}{|c|}{ Sextupole $\left(1 \times 10^{-4}\right)$} \\
\hline & \multicolumn{3}{|c|}{$30 \mathrm{~mm}$} & \multicolumn{3}{|c|}{$30 \mathrm{~mm}$} & \multicolumn{3}{|c|}{$30 \mathrm{~mm}$} \\
\hline & Sys & Ran & $\Phi\left({ }^{\circ}\right)$ & Sys & Ran & $\Phi\left(^{\circ}\right)$ & Sys & Ran & $\Phi\left({ }^{\circ}\right)$ \\
\hline 1 & 0 & 0 & 0 & 0 & 0 & 0 & 0 & 0 & 0 \\
\hline 2 & 0 & 3 & 0 & 0 & 3 & 0 & 0 & 0 & 0 \\
\hline 3 & 3 & 1 & 0 & 1 & 1 & -80 & 0 & 20 & 0 \\
\hline 4 & 0 & 1 & 0 & 1 & 1 & 150 & 1 & 3 & -85 \\
\hline 5 & 3 & 1 & 0 & 1 & 1 & 80 & 1 & 1 & -130 \\
\hline 6 & 0 & 1 & 0 & 1 & 1 & 0 & 1 & 1 & -15 \\
\hline 7 & 3 & 1 & 0 & 1 & 1 & 180 & 1 & 1 & 66 \\
\hline 8 & 0 & 0 & 0 & 1 & 1 & 5 & 1 & 1 & 203 \\
\hline 9 & 0 & 0 & 0 & 1 & 1 & 75 & 1 & 1 & 1 \\
\hline 10 & 0 & 0 & 0 & 1 & 1 & 180 & 1 & 1 & -116 \\
\hline 11 & 0 & 0 & 0 & 1 & 1 & 10 & 1 & 1 & 46 \\
\hline 12 & 0 & 0 & 0 & 1 & 1 & 180 & 1 & 1 & 84 \\
\hline 13 & 0 & 0 & 0 & 1 & 1 & 110 & 1 & 1 & -291 \\
\hline 14 & 0 & 0 & 0 & 1 & 1 & 25 & 1 & 1 & -10 \\
\hline 15 & 0 & 0 & 0 & 1 & 1 & 0 & 1 & 1 & -182 \\
\hline 16 & 0 & 0 & 0 & 1 & 1 & 0 & 1 & 1 & 0 \\
\hline
\end{tabular}

accelerator toolbox [16]; the dynamic aperture with energy spread up to $\pm 1 \%$ can be obtained. Compared with the dynamic aperture without magnet errors, the dynamic aperture with magnet errors decreased about $20 \%-40 \%$, which is shown in Figs. 8-10, for three different momentum compaction factor modes. In these figures, the blue line is 3 times injected positron bunch size which is also the lower limit of the dynamic aperture, and the red line is the dynamic aperture obtained by tracking with magnet errors, modified by taking into account, analytically, the reduction due to the nonlinear wiggler effects [17].

To get the damping ring lattice with momentum compaction as $2 \times 10^{-4}$, strong quadrupoles are needed in the arc cell to decrease the horizontal dispersion function. That has two consequences: first, the natural chromaticity is increased; second, the chromaticity correction sextupole's strength is increased also due to the decreased horizontal dispersion. These issues will make the dynamic aperture optimization much harder. Using the techniques described before, the dynamic aperture of the $2 \times 10^{-4}$ momentum compaction mode is optimized, as shown in Fig. 10. The corresponding FMA results are shown in Figs. 11 and 12.

The two groups of sextupoles in the arc cell have already corrected the first-order chromaticity to zero, so the remained tune variation with energy spread is from the second-order or higher-order chromatic effects. To further
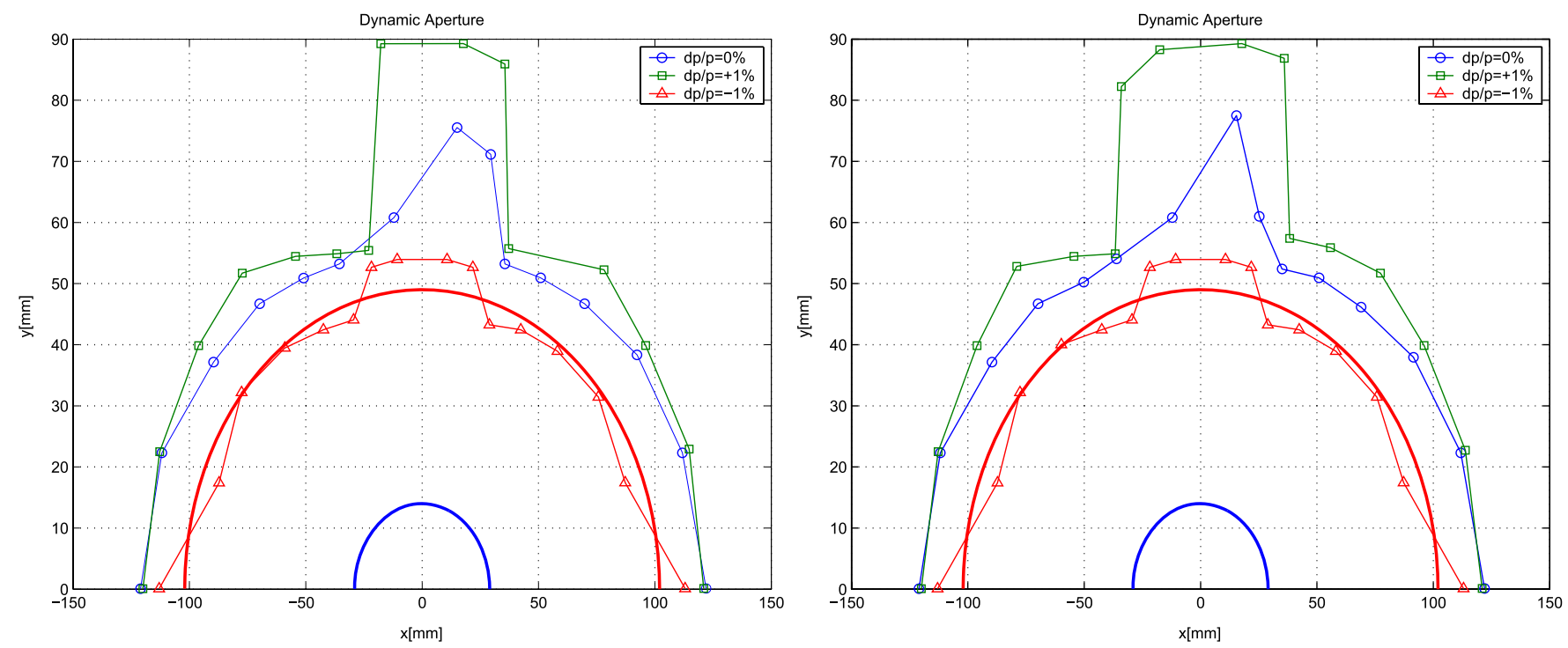

FIG. 8. (Color) Dynamic aperture for the mode with momentum compaction $6 \times 10^{-4}$, without errors (left) and with errors (right). 

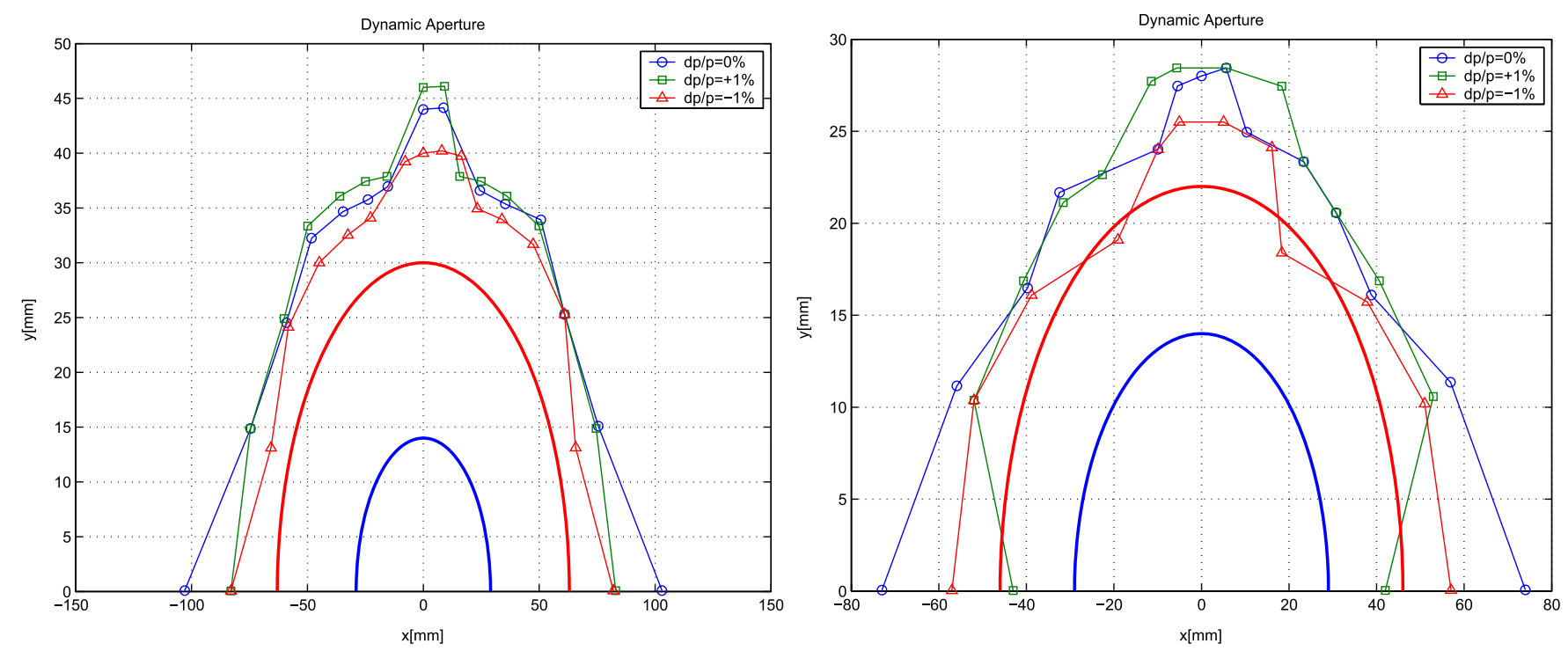

FIG. 9. (Color) Dynamic aperture for the mode with momentum compaction $4 \times 10^{-4}$, without errors (left) and with errors (right).

improve the dynamic aperture of the mode with momentum compaction $2 \times 10^{-4}$, we try the harmonic sextupole technique to correct the high-order harmonic's influence on the dynamic aperture caused by the sextupoles in the arc section. The harmonic sextupoles are placed in the sections where it is horizontal dispersion free, in order not to influence the first-order chromaticity. Meanwhile, the beta functions and the phase advance at these harmonic sextupoles should be carefully selected.

The mode with momentum compaction $2 \times 10^{-4}$ and the mode with momentum compaction $4 \times 10^{-4}$ are first analyzed in the accelerator code COSY [18]. Then three groups of harmonic sextupoles are added in the lattice with the momentum compaction $2 \times 10^{-4}$. One group (four sextupoles) is placed at the location where the horizontal beta function is larger and the vertical beta function is smaller, and that is in the two drifts of the injection/ extraction sections. The second group (eight sextupoles) is placed at the location where the horizontal beta function is smaller and the vertical beta function is larger (near the quadrupole at the exit of the dispersion suppressor). The last group is placed at the match section between the arc section and the rf section, where the horizontal and vertical beta functions are equal. The optimized dynamic aperture with harmonic sextupoles is much better than the one without, as shown in Fig. 13. To further improve the dynamic aperture, a full scan of the harmonic sextupole strength is still underway.
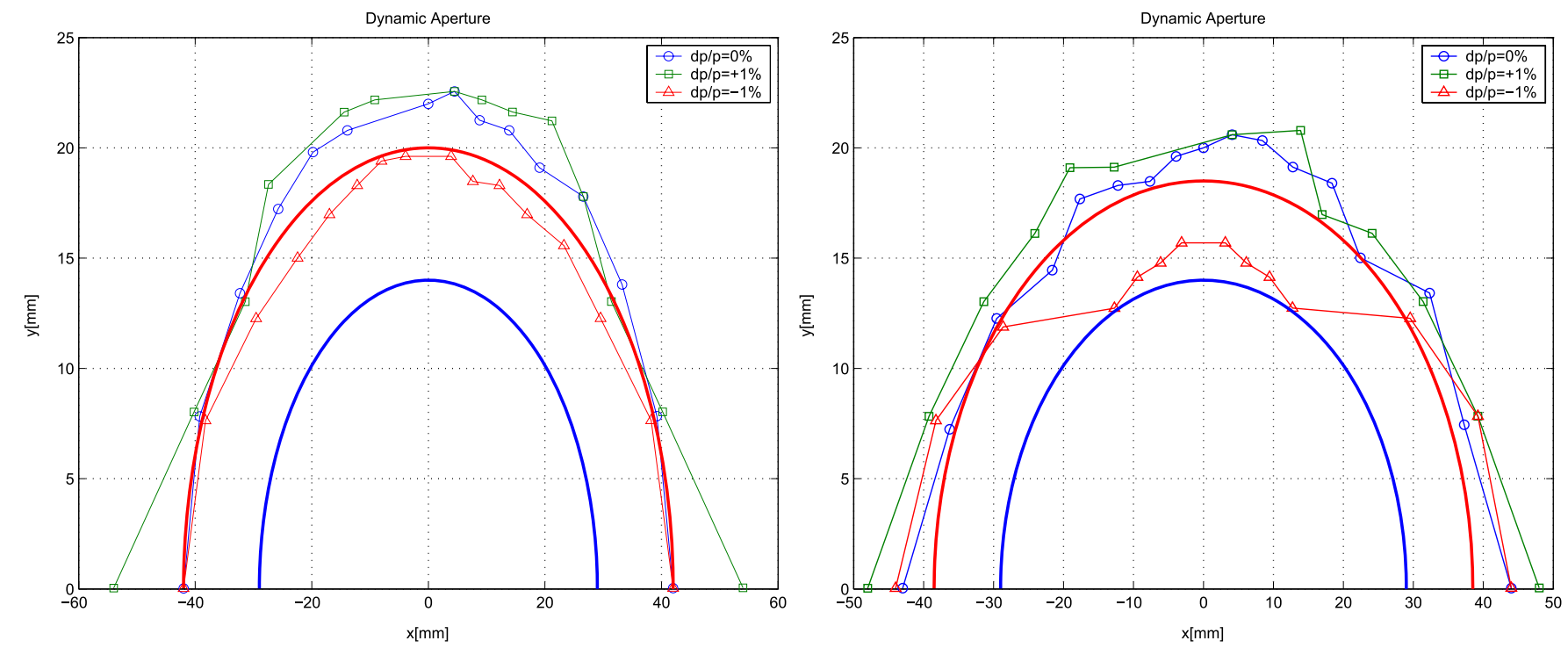

FIG. 10. (Color) Dynamic aperture for the mode with momentum compaction $2 \times 10^{-4}$, without errors (left) and with errors (right). 


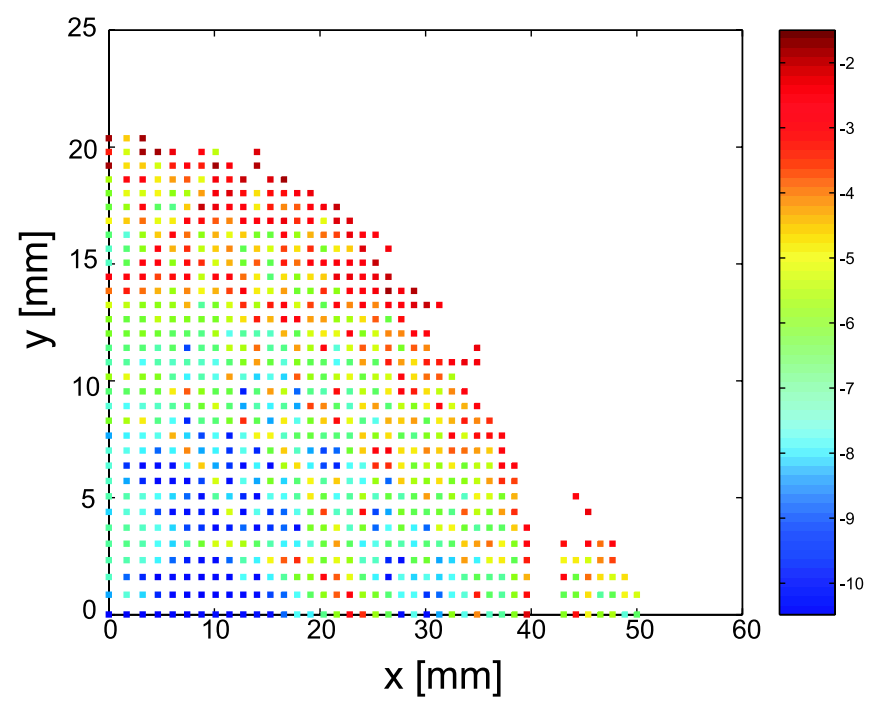

FIG. 11. (Color) Dynamic aperture with FMA, for the mode with momentum compaction $2 \times 10^{-4}$.

\section{TOUSCHEK LIFETIME}

The positron and electron beams will be stored in the damping ring for $200 \mathrm{~ms}$ in regular operation. However, a very short lifetime will cause problems while commissioning the damping ring [9]. As the Touschek lifetime is measured in minutes or hours, we shall make an estimate of the Touschek lifetime in our damping ring lattice. The Touschek lifetime estimation formulas valid for flat beams and nonrelativistic energies in the rest frame of the beam are given by [19]

$$
\frac{1}{\tau}=\frac{r_{e}^{2} c N_{0}}{8 \pi \gamma^{2} \delta_{\max }^{3} \sigma_{x} \sigma_{y} \sigma_{z}} D(\varepsilon),
$$

where $r_{e}$ is the electron radius, $c$ is the speed of light, $N_{0}$ is

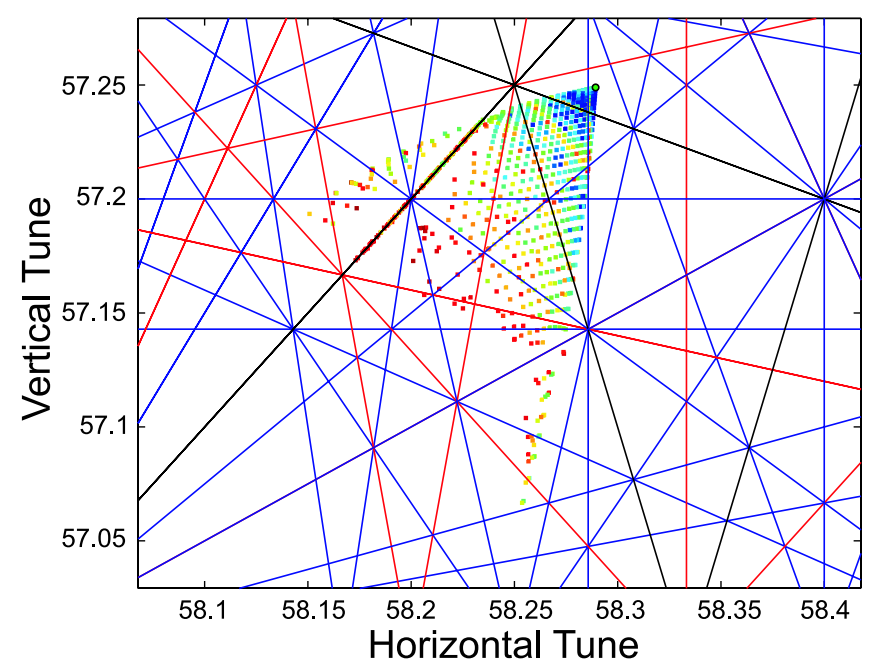

FIG. 12. (Color) Footprint on the tune space, for the mode with momentum compaction $2 \times 10^{-4}$.

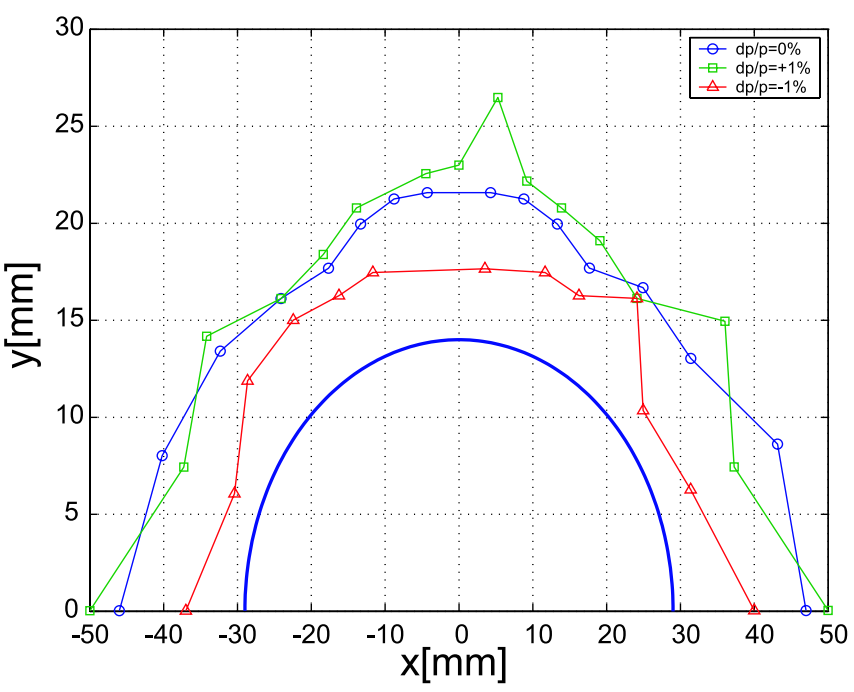

FIG. 13. (Color) Optimized dynamic aperture with harmonic sextupoles (with errors), for the mode with momentum compaction $2 \times 10^{-4}$.

the number of particles per bunch, $\delta_{\max }$ is the energy acceptance, $\gamma$ the relativistic factor, and $\sigma_{x}, \sigma_{y}$, and $\sigma_{z}$ are the horizontal, vertical, and longitudinal rms bunch sizes. The function $D(\varepsilon)$ is given by

$$
\begin{aligned}
\frac{2 D(\varepsilon)}{\sqrt{\varepsilon}}= & -3 e^{-\varepsilon}+\varepsilon \int_{\varepsilon}^{\infty} \frac{\ln u}{u} e^{-u} d u+(3 \varepsilon-\varepsilon \ln \varepsilon+2) \\
& \times \int_{\varepsilon}^{\infty} \frac{e^{-u}}{u} d u
\end{aligned}
$$

and the parameter $\varepsilon$ is given by

$$
\varepsilon=\left(\frac{\beta_{x} \delta_{\max }}{\gamma \sigma_{x}}\right)^{2}
$$

where $\beta_{x}$ is the horizontal beta function.

Taking the rf acceptance of the damping ring mode with momentum compaction $4 \times 10^{-4}$, which is $1.48 \%$ as listed in Table I, we can estimate the Touschek lifetime by using formula (6). While the electrons per bunch are assumed to be $2 \times 10^{10}$, the Touschek lifetime is calculated to be around 160 minutes.

\section{LATTICE FLEXIBILITY}

Recently, the requirements of reducing the momentum compaction range to have a smaller bunch length of $6 \mathrm{~mm}$ (with similar or even smaller rf voltage) are raised by the damping ring group. Also locating the wiggler section and rf cavity section together in one straight, like in the above design, may cause radiation problems on the vacuum elements of the ring.

Based on these considerations, the layout of the above FODO lattice is changed from 4-folder symmetry to 8folder symmetry, which is similar with the baseline design. There are in all eight straight sections and eight arc sec- 
tions, among which four will be used to accommodate damping wigglers alone, and the other four for injection/ extraction and rf cavity. The linear optics is easily adjusted with slightly modified arc cells and other sections. The parameters are similar to the ones listed in Table I, and the dynamic aperture is large enough for injection from the positron source.

\section{SUMMARY AND CONCLUSION}

In summary, the advantages of this FODO damping ring lattice over the baseline design OCS8 [10] which is based on TME arc cells are as follows: smaller number of quadrupoles and sextupoles used (roughly two-thirds), and lower cost; freely tunable momentum compaction factor in the range between $2 \times 10^{-4}$ and $6 \times 10^{-4}$; better dynamic aperture; simpler layout, with only two wiggler sections and cryogenics shaft.

The disadvantage is that, as the wigglers are all placed at only two straight sections, the radiation power should be carefully treated in order not to have any damage from it.

In this paper, the overall design of the ILC damping ring lattice based on modified FODO arc cells is presented, including the various considerations and comparisons, the layout of the ring, the optics design of the subsections, the chromaticity correction and dynamic aperture optimization with high-order magnet errors and nonlinear wiggler effects included. Also, frequency map analysis, harmonic sextupoles, and other useful techniques are used for the optimization of the lattice design and the dynamic aperture promotion. Touschek beam lifetime has been evaluated. The comparison with the ILC baseline damping ring lattice design is made in the summary section. Studies on this damping ring lattice design are still ongoing.

\section{ACKNOWLEDGMENTS}

The author would like to thank A. Xiao and L. Emery at ANL for the optics design of the rf/wiggler section. Thanks also go to Professor Mike Zisman at LBNL who gave many useful suggestions and comments. This work was supported by the National Natural Science Foundation of China under Grants No. 10525525 and No. 10775154.
[1] T. O. Raubenheimer, L.Z. Rivkin, and R.D. Ruth, in Proceedings of Snowmass 1988, Snowmass, Colorado (World Scientific, Singapore, 1988), p. 620.

[2] T. O. Raubenheimer et al., SLAC Report No. SLACReport-474, 1996, edited by T. O. Raubenheimer.

[3] J. P. Potier and L. Rivkin, CERN Report No. CERN-PS97-020, 1997.

[4] W. Decking, Report No. TESLA 2001-11, 2001.

[5] P. Emma and T. Raubenhemier, Phys. Rev. ST Accel. Beams 4, 021001 (2001).

[6] S. Mishra et al., Report No. FERMILAB-TM-2272-ADTD, 2004.

[7] A. Xiao, An Alternative TESLA Damping Ring Lattice Design at Fermilab, Techical Report, 2004.

[8] Y. Cai, Report No. SLAC-PUB-11084, 2005; A. Wolski, Reports No. LBNL-57045, No. LBNL-56505, and No. SLAC-PUB-11208, 2005.

[9] A. Wolski, J. Gao, and S. Guiducci, Report No. LBNL59449, 2006.

[10] A. Xiao and L. Emery, Proceedings of PAC2007, Albuquerque, New Mexico, USA.

[11] L. C. Teng, Fermilab Report No. TM-1269, 1984.

[12] A.W. Chao and M. Tigner, Handbook of Accelerator Physics and Engineering (World Scientific, Singapore, 1998).

[13] ILC GDE, ILC Reference Design Report, 2007, http:// www.linearcollider.org/cms/.

[14] M. Palmer, Lattice Session Summary, internal report presented in the ILC Damping Rings R\&D Meeting, Frascati, Italy, 2007.

[15] H. Ehrlichmann, S. Guiducci, K. Kubo, M. Kuriki, and A. Wolski, Recommendations for ILC Configuration Satisfying Timing Constraints, internal note, 2006; also see G. Penn, Timing issues for the ILC damping rings, internal note, 2007; via https://wiki.lepp.cornell.edu/ilc/ bin/view/Public/DampingRings/WebHome.

[16] A. Terebilo, Report No. SLAC-PUB-8732, 2001.

[17] J. Gao, Nucl. Instrum. Methods Phys. Res., Sect. A 451, 545 (2000).

[18] M. Berz et al., COSY INFINITY, http://www.bt.pa. msu.edu/index_cosy.htm.

[19] H. Wiedemann, Particle Accelerator Physics II (Springer, New York, 1995), p. 329. 